\title{
Round Robin Load Balancer for the Three Node Swarm Cluster for Hosting a Service on the Cloud
}

\author{
Aaradhya Shukla, Ayush Singh Chauhan, J. Kalaivani
}

\begin{abstract}
In this project we will be creating an application which uses socket programming for communication and all of the data/metadata are saved in mongo $D B$. We will be taking this application and hosting it on a 3 node swarm cluster. This cluster will be using Docker swarm technology to create a private network through which each of the nodes can talk to each other along a specified RPC port. So, based on this concept we will be making a Docker container which will handle the execution of multiple processes which lead to working of different projects concurrently. Docker is a PC program that performs working framework level virtualization otherwise called containerization.
\end{abstract}

Keyword: project, programming for communication, called containerization.

\section{INTODUCTION}

These days, distributed computing is becoming the business membership to outer administrations in in so many sectors. Its guideline depends on the compensation for-use display that can influence diverse components, for example, the mentioned application, information stockpiling limit, memory handling and number of clients. The fundamental targets of this task are to manage and to setup: Receipt, in light of asset utilization so as to accomplish the business effectiveness of the organization; Avoid wastage of asset utilization and devour as it were what is vital and productive to applications to accomplish vitality effectiveness. Facilitate for the client the appropriation of the compartment's innovation to build up a commercial Centre. With regards to ongoing venture, we propose, in this paper, our underlying thoughts for another booking methodology executed in Docker Swarm, the principle innovation utilized in the venture. This paper is for the most part committed to usage subtleties and general thoughts. More work has been done for the current year to broaden this work, see for example and a general union of our system is at present under looking into. The Discussion area will clarify, last on, some situating actualities of this work contrasted with progressing works. Docker Swarm is a bunching and booking apparatus for Docker holders. It chooses the principal demand that must be executed by utilizing the traditional FIFO system. At that point, Docker Swarm picks the suitable machine for example fulfilling the need of the client as far as CPU centers, by utilizing one procedure among the accompanying methodologies: Our proposed financial model relies upon three SLA classes to answer to the customers' needs.

Revised Manuscript Received on February 05, 2020.

* Correspondence Author

Aaradhya Shukla, Dept of computer science and engineering, SRM IST, India. E-mail: aaradhya.shukla229@gmail.com

Ayush Singh Chauhan, Dept of computer science and engineering, SRM IST, India. E-mail: ayushsingh090@gmail.com

Dr. J. Kalaivani, Dept of Computer Science, SRM IST, India. E-mail: kalaivaj.srmist.edu.in

(C) The Authors. Published by Blue Eyes Intelligence Engineering and Sciences Publication (BEIESP). This is an open access article under the CC BY-NC-ND license (http://creativecommons.org/licenses/by-nc-nd/4.0/)
The showing with three classes is motivated by the mechanical endeavors and relies upon the discernment that encouraging courses of action don't allow makers or cloud providers to offer to their customers a sensible or exact receipt, for instance a precise receipt with reverence to the use of benefits by the referenced organization. Seen that the errand must respond to the going with use cases concerning the sent organizations, every organization addresses one SLA class Long administration it is a determined administration encountering pinnacles of burden (CPU, smash, plate, organize) like times of dormancy.

\section{RELATED WORK}

Previously similar kind of work has been done. This includes hosting services on the cloud. The basic cloud architecture in the older days used to use the Round Robin Load Balancer, which is nothing but an algorithm that is used to manage the client server request.

So all of the previous work using this Round Robin techniques were not so fast and were neither that efficient on providing the proper results.

Later On Click Load Balancer was introduced in the cloud system .This system is really effective and it helps in reaching parallel computing and simultaneous request handling .The bad aspect to this technology is that it is very costly method to implement.

So in order to facilitate this method and make it more refine and less expensive to the the majority of people we decided to use the Docker Swarm technology .]. Docker is stage for structure, dispatching and running applications. It doesn't contain the entire working framework, however simply the important parallels and libraries. It fundamentally encapsulates a run time condition. It is kept running on client space over the OS. There is no understanding of hypervisor. Namespaces encourage the compartment to have its very own system, IP and so forth. Control bunches deal with the utilization of memory and CPU. It is a stage for creating, delivering and running applications. It is somewhat similar to the virtual machine. However, the entire OS isn't made. Docker utilizes the host machine's piece. It is lightweight and decreases the size. The host machine is of no worry. Pictures can be pulled from the stores or can be made by clients (for example by utilizing Dockerfiles). Dockerfiles can be utilized to assemble pictures. Docker libraries are used to store the pictures. Docker compartments get made when pictures are run. Information volumes can likewise be shared between compartments. The compartments/microservices (benefits that speak with each over a system (administration arranged engineering)) can be associated with one another 


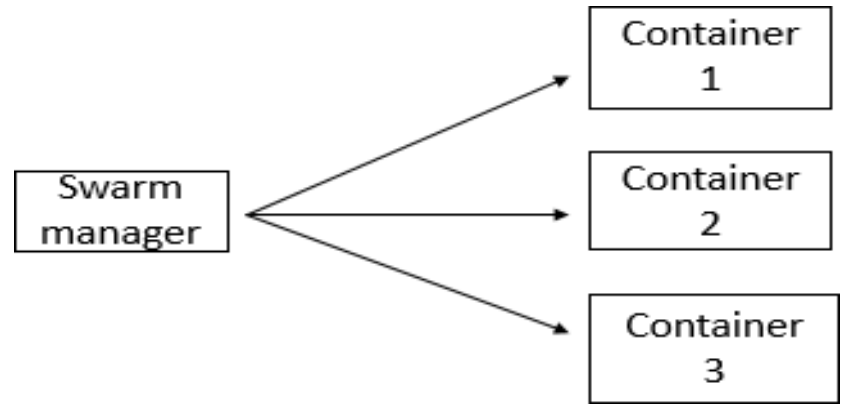

Figure 1:Basic Swarm container flow

Proposed improved planning calculation which depended on standard Max-Min calculation for burden balance in the flexible cloud. In this work they kept up an assignment status table to store the assessed continuous heap of VMs proposed in this paper and rough fulfilment time of undertakingsrequires timely re-service like laundry service.

\section{PROPOSED METHODOLOGY}

A.

A backend network model of the proposed system

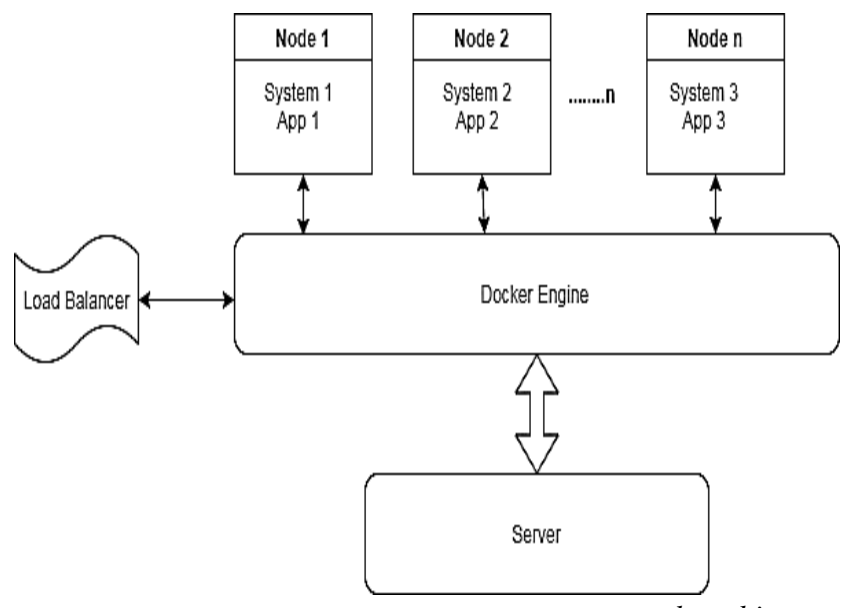

network architecture

Creation of an application which will be hosted on the cloud.We will be using Docker swarm and Routing mesh Technology to create a private network all of this together will act as a load balancer. We will be using 4 containers and in which two of them will act as a swarm container which in turn can create and delete requests.

Proposed framework will give you the opportunity to pick the information of any subject.Here, it gives you the effect the outcomes and measurements will have on the separate field.Proposed system.

\section{B. Cloud connectivity and computing:}

All the requests of clients will be in cloud and all the database used for storing the other meta data are stored and computed in MongoDb The Cloud service is encapsulated with layers of security protecting it from potential attack. The personal details are private and order details are only shown to the client it is assigned to. All the data is resided in distributed servers and retrieved only when queried.

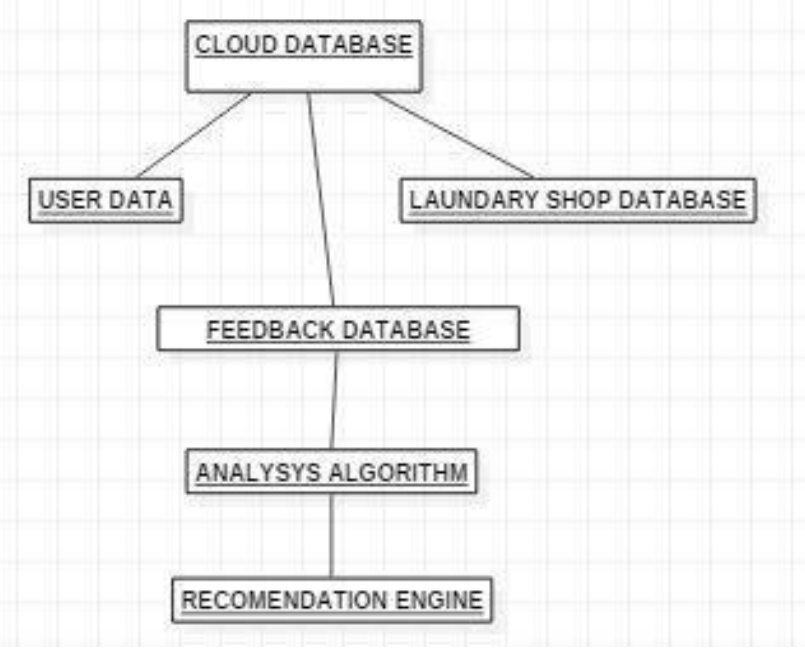

Figure 2: application's cloud architecture

\section{VM Engine:}

Its job is to maintain a sub-level between the system/server, so as to provide seamless dataflow in the given model. It maintains all the virtual nodes and keeps them at a separate level-1. It provides the start for the virtual machine and also maintains the cloud architecture in-order ton ensure security protocols between server and n-node user.

\section{Resource Allocator:}

with This part manages resource allocation in the system, as many virtual servers try to access the system it will create different allocation times based on priority. It is a very important component of the model as it will help manage the resources that might try to enter the critical section at once.

\section{E. System Manager:}

It will overlook the complete model. It can be considered as the master component of the model because everything has to pass through the system manger. It will keep in check if each and every module is working properly because if it isn't it could create large security breaches which will make it difficult for the system administrator to manage the virtual network.

\section{F. Load Balancer:}

Its job will be to avoid deadlock after the resources have been allocated. It will help decrease the starvation through context switching which will help in the prevention of deadlock as it will not allow a virtual node-n to access the Critical section for a long period of time.

\section{RESULT ANALYSIS}

We used Digital Ocean a Cloud Service hosting platform for creating linux virtual servers. 
These servers are referred to as droplets.We used for Droplets among which one was made as the swarm manager .With the help of visual studio we loaded the Docker Script into each of the nodes thereby making 3 nodes as workers and 1 as the manager.All of these containers communicated together using routing mesh technique frequently establishing RPC ports for inner and outer communication.Using this we played our voting application over it. The security was through the linux 'ssh' command.The resultant was a full proof and secured voting system.

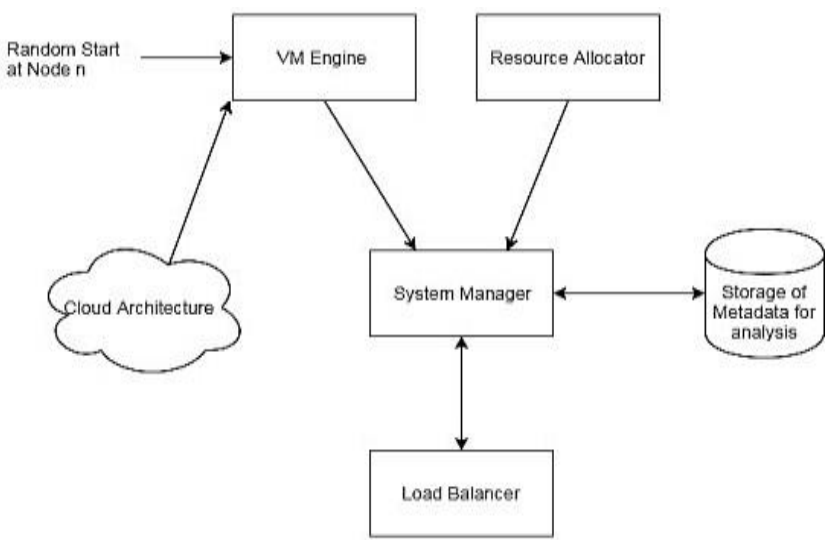

Figure 3:overall system architecture

\section{CONCLUSION:}

Using technologies like Docker and Docker swarm we were able to deploy a standard voting application to a 3 node swarm cluster where each node can be either a swarm manager or a swarm worker and communicating among each other via an open RPC port. Thus we created a load balancer for our chat application. The implementation part included two sets of our project backend and frontend.

\section{REFERENCES:}

1. G. Rastogi and D. R. Sushil, "Analytical Literature Survey on Existing Load Balancing Schemes in Cloud Computing," International Conference on Green Computing and Internet of Things, 2015

2. B. B. Rad, H. J. Bhatti and M. Ahmadi, "An Introduction to Docker and Analysis of its Performance," International Journal of Computer Science and Network Security, vol. 17, no. 3, pp. 228- 235, 2017.

3. C. Cerin, "A New Docker Swarm Scheduling Strategy," Universite de Paris.

4. K. S. Akshay, K. J. Anish, K. Amit, K.

5. T. Vivek and H. Praveen, "RESOURCE MONITORING OF DOCKER CONTAINERS," International Journal of Advance Engineering and Research Development.

6. S. S. Hegde and D. P. Jayarekha, "Basic Analysis of Docker Networking," International Journal of Advanced Research in Computer Science, vol. 8, no. 5, pp. 740-743, 2017.

7. P. J. Dilip and P. K. Nangana, "Docker, An Optimized Virtualization Using Container," International Journal for Research in Applied Science \& Engineering Technology.

8. P. Dziurzanski and L. S. Indrusiak, Value-Based Allocation of Docker Containers.

9. The Docker Book | John Martin and Jmaes Turbull ,2016 publications

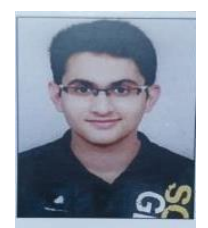

\section{AUTHORS PROFILE:}

Aaradhya Shukla, is presently doing is Computer Science and Engineering from SRM IST Chennai. His Hometown is in Lucknow and he did his Class 10th and 12th from ST. Francis College, Lucknow,Uttar Pradesh. Aaradhya shukla has earlier worked as an intern in E, soft. ConnectHe has previously worked on Android Projects. Aaradhya Shukla always had a great interest in Cloud Computing .This is the first time that he has Worked on a research paper. Aaradhya Shukla will be joining TCS, as a Software Engineer in July . Aaradhya Shukla has worked on several other projects That were based on android application development .

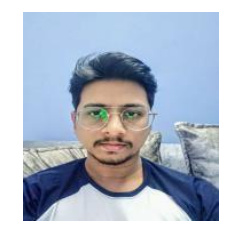

Ayush Singh Chauhan. is presently doing Computer Science and Engineering from SRM IST Chennai. Ayush Singh Chauhan hails from Lucknow And he has done his class 10th and 12th from CMS Aliganj Lucknow. Ayush Singh Chauhan has Done couple of internships based on web dev- -velopment .Ayush always had a keen interest In Networking and Big Data. He is applying for Foreign Universities for His masters in Big Data .Ayush and Aaradhya started working on this pro- -ject when they were in third year. Ayush has previously worked on several Cloud projects

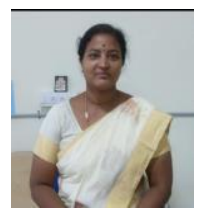

Dr. J. Kalaivani (Jeyaraj Kalaivani) obtained her Bachelor's Degree in ComputerScience and Engineering, National Engineering College, Anna University, Tamil Nadu, India in2005. Master's Degree in Computer Science and Engineering, Mepco Schlenk EngineeringCollege, Anna University, Tamil Nadu, India in 2008. Ph.D. in the Department of InformationTechnology, Madras Institute of Technology, Anna University, Chennai, Tamil Nadu, India in2017. She is working as Assistant Professor in the Department of Computer Science andEngineering, SRM Institute of Science and Technology, Kattankulathur, Kanchipuram District,Tamil Nadu, India. Her research interest is Computer Networks 